\title{
Qualidade de Vida de Acadêmicos de Medicina: Há Mudanças durante a Graduação?
}

\section{Quality of Life of Medical Students: Are There Changes during Undergraduate Training?}

\author{
Aline Maria Fatel da Silva Pires ${ }^{I}($ [ \\ Waléria Dantas Pereira Gusmão ${ }^{I, I I}$ \\ Lucyo Wagner Torres de Carvalho ${ }^{I}(\mathbb{D}$ \\ Monique Maria Lucena do Suruagy do Amaral ${ }^{I I}$ (D)
}

PALAVRAS-CHAVE

- Qualidade de Vida.

- Saúde.

- Estudantes de Medicina
${ }^{\mathrm{I}}$ Universidade Estadual de Ciências da Saúde de Alagoas, Maceió, Alagoas, Brasil.

${ }^{\text {II } C e n t r o ~ U n i v e r s i t a ́ r i o ~ C e s m a c, ~ M a c e i o ́, ~ A l a g o a s, ~ B r a s i l . ~}$ 


\section{KEYWORDS}

- Quality of Life.

- Health.

- Medical Students.

Recebido em 13/6/20

Aceito em 6/8/20

\section{INTRODUÇÃO}

O conceito de qualidade de vida foi incialmente enquadrado como um complemento ao conceito de saúde ${ }^{1}$. Embora não exista, ainda, uma definição para o que seja qualidade de vida, o conceito mais aceito na atualidade foi o proposto pelo grupo World Health Organization Quality of Life (Whoqol) da Organização Mundial de Saúde (OMS). Para esse grupo, qualidade de vida consiste na percepção do indivíduo de sua posição na vida, no contexto da cultura e sistema de valores nos quais ele vive e em relação aos seus objetivos, expectativas, padrões e preocupações ${ }^{2-4}$. Essa definição direcionou a criação de um instrumento confiável que avaliasse internacionalmente a qualidade de vida ${ }^{3}$.

Assim, com a colaboração de diversos países, a OMS desenvolveu o questionário World Health Organization Quality of Life (Whoqol-100), que permite analisar o quanto diferentes aspectos da vida podem ser satisfatórios ou não para o indivíduo numa perspectiva transcultural ${ }^{1,4,5}$. O instrumento Whoqol foi traduzido para o português por um grupo de pesquisadores da Universidade Federal do Rio Grande do Sul (UFRGS) ${ }^{2}$.

A sociedade contemporânea define metas e padrões de vida a serem alcançados, seja de forma consciente ou não. Isso se dá por meio de processos de renovação e transmissão cultural que se incorporam na percepção e expectativa dos sujeitos perante a própria vida, o que causa uma grande procura por profissões que permitam ser reconhecidos, bemsucedidos e remunerados ${ }^{6}$. A preocupação com a qualidade e o padrão de vida é uma questão que repercute para o indivíduo e para a sociedade. Além disso, historicamente, o curso da saúde de maior impacto e retorno social é a graduação em Medicina.

Entretanto, o status e o ganho financeiro que cercam essa profissão nem sempre vêm acompanhados de uma boa qualidade de vida. O médico se

\section{ABSTRACT}

Introduction: The concept of health has been broadened beyond the absence of disease and now involves all and value system under which they live. Method: The objective of this study was to analyze the quality of life of medical students at the State University of Health Sciences of Alagoas (UNCISAL), through the abbreviated World Health Organization Quality of Life (WHOQOL-BREF) questionnaire. The BREF contains 26 questions, two of which are general about quality of life and health satisfaction, and 24 are divided into domains: physical, psychological, social relations and environment. Responses are scored from one to five, by degree of intensity Once the raw data had been collected, mean scores and domains were calculated and converted on to a scale from 0 to 100. Results: Of the 188 students, $62.03 \%$ considered their quality of life good or very good, while $54.25 \%$ reported being neither satisfied nor dissatisfied, dissatisfied or very dissatisfied with their health. (he domains none of the groups attained the "region of success", with a scores over the course of the training is observed. First and second-year students were evaluated worse in the domains, showing that anxiety and expectation about undergraduate training can negatively affect quality of life. Regarding the domains, "sleep and rest" stands out as the worst evaluated issue, occupying the "region of failure", followed by the question that evaluates the opportunity for leisure and recreation, probably due to the exhausting training also harming students' physical, mental and emotional health. Regarding gender, women's assessment was lower in all domains and general issues, with significant differences in the psychological domain $(p=0.04)$ and health satisfaction $(p=0.04)$. Conclusion: It is necessary to develop interventions capable of providing support to medical students, helping them to cope with the difficulties they will face during the course.

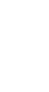
. 
pública localizada em Maceió, no estado de Alagoas. Em 2017, a universidade possuía um total de 300 estudantes de Medicina matriculados, divididos do primeiro ao sexto ano. O estudo foi aprovado pelo Comitê de Ética em Pesquisa sob parecer no 68885417.1.1001.0039 e recebeu apoio financeiro da Fundação de Amparo à Pesquisa do Estado de Alagoas (Fapeal).

A coleta foi realizada de agosto a dezembro de 2017 utilizando o instrumento de avaliação de qualidade de vida abreviado, o WhoqolBref. Esse questionário foi desenvolvido a partir do Whoqol-100, na tentativa de permitir que o instrumento fosse respondido em um menor tempo, embora mantivesse as mesmas características psicométricas do instrumento original ${ }^{4,5}$. Tem 26 questões, das quais 24 estão distribuídas em quatro domínios: físico (sete questões: Q3, Q4, Q10, Q15, Q16, Q17, Q18), psicológico (seis questões: Q5, Q6, Q7, Q11, Q19, Q26), relações sociais (três questões: Q20, Q21, Q22) e meio ambiente (oito questões: Q8, Q9, Q12, Q13, Q14, Q23, Q24, Q25). As outras duas são perguntas gerais sobre a qualidade de vida global (Q1) e a percepção geral do indivíduo em relação à saúde $(\mathrm{Q} 2)^{4,5,13,14}$, e não estão incluídas no cálculo dos domínios, sendo analisadas separadamente ${ }^{13,14}$. $\mathrm{O}$ instrumento leva em conta os últimos 15 dias vividos pelo indivíduo ${ }^{10,14}$ e necessita de aproximadamente dez minutos para ser respondido ${ }^{10}$.

Os questionários foram identificados a partir das iniciais do nome do participante, do sexo e do ano em que estava matriculado em 2017. Com os discentes do primeiro ao quarto ano, o instrumento foi aplicado em sala de aula, tendo autorização da coordenação do curso de Medicina da instituição e do docente responsável pela aula no momento da coleta. Já com os alunos do quinto e do sexto ano, por estarem pouco na instituição, já que passa a maior parte do tempo em ambiente hospitalar e de atendimento ambulatorial, o Whoqol-Bref foi digitalizado e compartilhado de forma gratuita, por meio de mídias sociais, com os estudantes do internato. $\mathrm{O}$ questionário on-line seguiu o mesmo procedimento do escrito, sendo apenas adaptado para o ambiente virtual e tendo a aceitação do Termo de Consentimento Livre e Esclarecido (TCLE) como pré-requisito para o seu preenchimento, como ocorreu com os que responderam ao questionário físico.

No Whoqol-Bref, as respostas são pontuadas de um a cinco na escala Likert, por grau de intensidade, conforme a metodologia do teste ${ }^{10,14}$. As questões Q3, Q4 e Q26 possuem escala invertida, de modo que quanto maior a pontuação, pior é a avaliação no quesito.

Finalizada a coleta, os valores dos domínios individuais dos sujeitos da pesquisa e as médias gerais das questões foram calculados por meio do programa proposto por Pedroso et al. ${ }^{13}$, o qual possibilita a utilização do software Microsoft Excel para o cálculo de escores e estatística descritiva do Whoqol-Bref. A validação desse aplicativo se deu após comparação com análises realizadas pelo software estatístico Statistical Package of Social Sciences (SPSS), programa originalmente proposto pela OMS para a realização da estatística do Whoqol-Bref, obtendo-se resultados idênticos. A ferramenta adaptada em Microsoft Excel é de mais fácil execução, de menor complexidade e de ampla difusão, o que facilita a consolidação e interpretação estatística, diminuindo a margem de erro. Com a pontuação bruta de um a cinco, calculam-se as médias dos escores das questões e dos domínios, os quais são convertidos em uma escala de 0 a 100.

A amostragem foi dividida em três partes: ciclo básico (primeiro e segundo anos), ciclo profissionalizante (terceiro e quarto anos) e internato (quinto e sexto anos), levando em consideração a proximidade de disciplinas e o estresse a que estão submetidos. Excluíram-se dois estudantes, uma vez que deixaram mais de duas questões de um mesmo domínio sem resposta, conforme orienta o Whoqol-Bref.

No geral, utilizaram-se o teste de Lilliefors para verificar normalidade e o teste de Levene para verificar homoscedasticidade, que são pressupostos de parametria. Para comparação dos ciclos quando atendidos os pressupostos, utilizaram-se a ANOVA e o teste post hoc Tukey; quando não atendidos, utilizaram-se o teste de Kruskal-Wallis e teste post hoc de Dunn.

Já na comparação entre os sexos em relação aos domínios, quando atendidos os pressupostos, utilizou-se teste $\mathrm{t}$ de Student; quando não atendidos, adotou-se o teste de Mann-Whitney. O alfa foi 0,05, e utilizouse o programa Bioestat 5.0 para as comparações.

Conforme exemplo proposto por Saupe et al. ${ }^{14}$, em que se consideram os valores entre 0 e 40 como "região de fracasso", 41 e 70 como "região de indefinição" e acima de 71 como "região de sucesso", analisaram-se os resultados com base nessa classificação.

\section{RESULTADOS}

Dos 300 discentes de Medicina matriculados na instituição no ano de 2017, 190 responderam ao Whoqol-Bref, mas dois alunos foram excluídos da amostra, totalizando 188 estudantes do primeiro ao sexto ano. Destes, 65 (34,57\%) são do primeiro e segundo anos; 77 (40,95\%), do terceiro e quarto anos; e 46 (24,46\%), do quinto e sexto anos. Havia $84(44,68 \%)$ indivíduos do sexo masculino e $104(55,31 \%)$ do sexo feminino.

Quanto à primeira questão do Whoqol-Bref - "Como você avaliaria a sua qualidade de vida?" -, numa escala de 1 a 5 , os alunos do meio do curso apresentaram melhor resultado, mas não houve diferença significativa $(\mathrm{p}=0,84)$ : 3,67 (terceiro e quarto anos) versus 3,65 (primeiro e segundo anos) versus 3,52 (quinto e sexto anos). Já em relação à segunda questão - "Quão satisfeito(a) você está com a sua saúde?” -, os alunos do início do curso tiveram melhor avaliação, mas também não houve diferença estatisticamente significante $(\mathrm{p}=0,59): 3,34$ (primeiro e segundo anos) versus 3,23 (terceiro e quarto anos) versus 3,20 (quinto e sexto anos). Convém ressaltar que 62,03\% dos estudantes de Medicina consideraram a sua qualidade de vida "boa" ou "muito boa", enquanto 54,25\% estavam "nem satisfeitos nem insatisfeitos", "insatisfeitos" ou "muito insatisfeitos" com sua saúde.

Já em relação ao sexo, as mulheres, numa escala de 1 a 5 , tiveram a pior avaliação quanto à qualidade de vida $(3,57$ versus 3,67$)$ e à satisfação com a saúde $(3,08$ versus 3,44$)$, embora tenha havido diferença significativa apenas no grupo do internato $(\mathrm{p}=0,04)$, em relação à satisfação com a saúde.

$\mathrm{Na}$ análise das médias gerais dos escores das questões (Gráfico 1), destaca-se o "sono e repouso" (Q16 = 39,97) como a questão de pior avaliação, ocupando a "região de fracasso", seguida da questão que avalia a oportunidade de lazer e recreação $(\mathrm{Q} 14=48,94)$. Como as de melhor avaliação, alcançando a "região de sucesso", destacam-se as questões que avaliam a capacidade do indivíduo de se locomover $(\mathrm{Q} 15=81,15)$, a autoestima (Q6 = 78,51) e a satisfação com as condições do local onde mora $(\mathrm{Q} 23=72,46)$.

Não houve diferença estatística, em relação aos grupos, nos domínios relações sociais, psicológico e meio ambiente. Já em relação ao domínio físico ( $\mathrm{p}=0,02)$, houve um aumento gradativo das médias durante o curso médico: 57,91 (primeiro e segundo anos) versus 60,04 (terceiro e quarto

REVISTA BRASILEIRA DE EDUCAÇ̃̃O MÉDICA

3 44 (4) : e124; 2020 


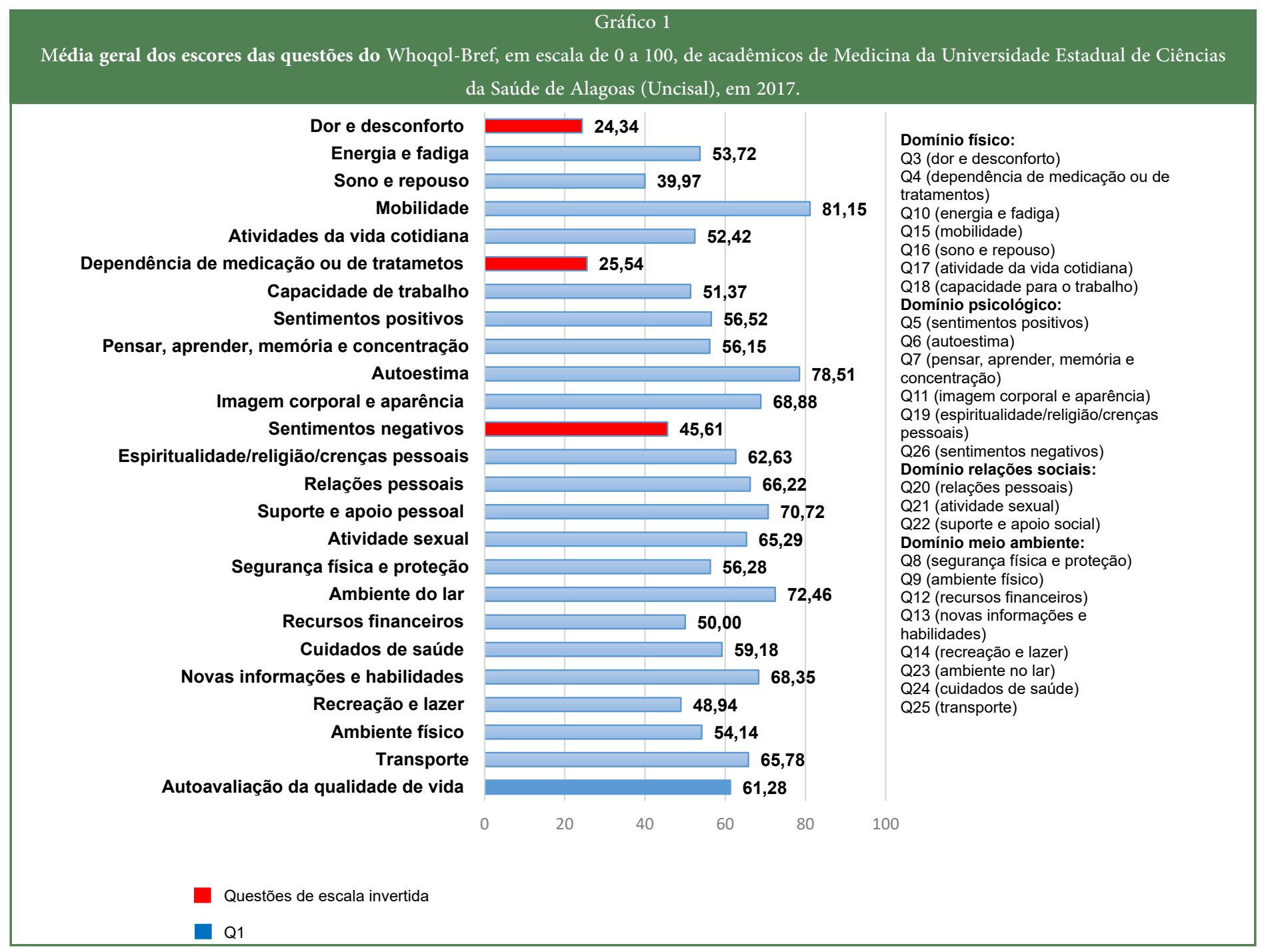

Fonte: Gráfico disponibilizado pela ferramenta de Pedroso et $\mathrm{al}^{13}$.

anos) versus 65,76 (quinto e sexto anos). Os alunos do primeiro e segundo anos obtiveram as piores médias em todos os domínios (Tabela 1).

Em relação ao sexo, não houve diferença estatisticamente significativa, exceto no domínio psicológico, no grupo dos alunos do quinto e sexto anos $(\mathrm{p}=0,04)$ (Tabela 2$)$.

\section{DISCUSSÃO}

Avaliou-se a qualidade de vida durante todo o curso de Medicina, representado pelos acadêmicos do ciclo básico (primeiro e segundo anos), ciclo profissionalizante (terceiro e quarto anos) e internato (quinto e sexto anos). Percebeu-se que os estudantes de Medicina da Uncisal consideram sua qualidade de vida "boa" ou "muito boa" (62,03\%), embora estejam "nem satisfeitos nem insatisfeitos", "insatisfeitos" ou "muito insatisfeitos" com a sua saúde $(54,25 \%)$, provavelmente pelo desgaste físico, emocional e mental do curso médico. Tudo isso recai sobre um paradoxo, pois aqueles que são formados para cuidar do outro parecem não ter condições para tal.

Como os valores dos escores variam de 0 a $100^{15}$, observou-se que a qualidade de vida, nos três grupos, não foi muito boa em nenhum dos domínios do Whoqol-Bref, pois as médias não ultrapassaram os 70 pontos. Bampi et al. ${ }^{7}$ realizaram estudo com 84 estudantes de graduação em Medicina da Universidade de Brasília (UnB) utilizando o mesmo instrumento e obtiveram resultados semelhantes, pois, na avaliação média dos quatro domínios, nenhum deles alcançou a região de sucesso (> 71 pontos).

O domínio relações sociais avalia a satisfação do indivíduo com suas relações pessoais, com o apoio social que recebe e com sua atividade sexual ${ }^{13}$, e obteve o melhor resultado nos três grupos, embora não haja diferença estatística entre eles. Entretanto, os discentes do ciclo inicial (primeiro e segundo anos) referiram avaliação mais baixa em comparação aos demais. Ramos-Dias et al. ${ }^{15}$ demonstraram que o domínio relações sociais, em alunos do primeiro ano de Medicina de uma universidade de Sorocaba, em São Paulo, foi o mais comprometido, o que pode estar relacionado ao período de adaptação desses indivíduos ao ambiente e à rotina acadêmica.

A ausência de apoio familiar e institucional pode prejudicar a qualidade de vida dos acadêmicos de Medicina ${ }^{11}$, contribuindo para o desenvolvimento de ansiedade, depressão e outras doenças mentais ${ }^{8,9,11}$. Felizmente, a questão mais bem avaliada nesse domínio, entre todos os grupos, foi a que estima o suporte e apoio social que o indivíduo recebe $(\mathrm{Q} 22=70,72)$.

Estudo semelhante realizado em Recife, em Pernambuco, com 370 discentes de Medicina demonstrou o domínio psicológico dos 


\begin{tabular}{l} 
Tabela 1 \\
Domínios físico, psicológico, relações sociais e meio ambiente do \\
Whoqol-Bref nos diferentes anos de graduação em Medicina da \\
Universidade Estadual de Ciências da Saúde de Alagoas (Uncisal), \\
\multicolumn{5}{c}{ em 2017.} \\
\\
Fediana \\
Físico
\end{tabular}

ANOVA; Tukey; DP = desvio padrão.

Fonte: Elaborado pelos autores.

estudantes do final do curso como o de pior escore avaliado em relação aos alunos do primeiro ano ${ }^{10}$. Cunha et al. ${ }^{11}$, utilizando o Whoqol-100, verificaram, numa amostra de 607 alunos de Medicina do primeiro ao sexto ano da Escola Paulista de Medicina da Universidade Federal de São Paulo (EPM-Unifesp), que os do último ano apresentaram, em todos os domínios, os menores escores em comparação aos primeiranistas, exceto no de espiritualidade/religião e crenças pessoais. Além disso, os autores evidenciaram um declínio do domínio psicológico ao longo dos anos de curso. Este estudo com acadêmicos da Uncisal, contudo, evidenciou que os estudantes do início do curso obtiveram a pior avaliação nesse domínio, seguidos dos alunos do meio do curso e, por fim, daqueles alunos do internato, mas não houve diferença estatisticamente significativa.

A expectativa dos estudantes do início do curso de começar uma nova etapa na vida é grande. Entretanto, a frustração diante da realidade da faculdade - já que entrar na universidade não confere um ritmo de vida mais tranquilo do que o vivenciado no período pré-vestibular ${ }^{4,7}-\mathrm{e}$ ainda a dificuldade em encontrar utilidade nos seus estudos - pois muitos não conseguem relacioná-los com a prática clínica - podem gerar um sentimento de ineficiência e prejudicar o desempenho do acadêmico ${ }^{16}$. Além disso, durante o último semestre antes da formatura, os acadêmicos sentem-se inseguros quanto ao seu futuro profissional e à sua inserção no mercado de trabalho, muitas vezes por considerarem seu conhecimento teórico-prático insuficiente ${ }^{16,17}$. Nesse período, ainda há a preocupação com a aprovação em programas de residência médica e com a possibilidade de eles se tornarem bons médicos, o que aumenta os níveis de estresse nesses indivíduos ${ }^{4,7,8}$.

\section{Tabela 2}

Domínios físico, psicológico, relações sociais e meio ambiente do Whoqol-Bref nos diferentes anos de graduação, comparados entre os sexos, do curso de Medicina da Universidade Estadual de Ciências da Saúde de Alagoas (Uncisal), em 2017

\begin{tabular}{|c|c|c|c|c|}
\hline Físico & & Escores & & \\
\hline $1^{\circ}$ e $2^{\circ}$ anos & Mediana & Média & DP & p-valor \\
\hline Masculino & 60,71 & 58,75 & 17,21 & 0,44 \\
\hline Feminino & 57,14 & 55,60 & 15,96 & \\
\hline \multicolumn{5}{|l|}{$3^{\circ}$ e $4^{\circ}$ anos } \\
\hline Masculino & 64,29 & 60,68 & 21,56 & 0,55 \\
\hline Feminino & 60,71 & 58,24 & 11,17 & \\
\hline \multicolumn{5}{|l|}{$5^{\circ}$ e $6^{\circ}$ anos } \\
\hline Masculino & 67,86 & 69,87 & 14,32 & 0,06 \\
\hline Feminino & 60,71 & 61,65 & 15,39 & \\
\hline \multicolumn{5}{|l|}{ Psicológico } \\
\hline $1^{\circ}$ e $2^{\circ}$ anos & Mediana & Média & DP & p-valor \\
\hline Masculino & 62,50 & 58,47 & 23,76 & 0,84 \\
\hline Feminino & 57,14 & 59,49 & 16,20 & \\
\hline \multicolumn{5}{|l|}{$3^{\circ}$ e $4^{\circ}$ anos } \\
\hline Masculino & 66,67 & 64,90 & 17,65 & 0,33 \\
\hline Feminino & 62,50 & 61,43 & 14,22 & \\
\hline \multicolumn{5}{|l|}{$5^{\circ}$ e $6^{\circ}$ anos } \\
\hline Masculino & 66,67 & 68,12 & 13,33 & 0,04 \\
\hline Feminino & 62,50 & 59,42 & 15,45 & \\
\hline \multicolumn{5}{|l|}{ Relações sociais } \\
\hline $1^{\circ}$ e $2^{\circ}$ anos & Mediana & Média & DP & $\mathrm{p}$-valor \\
\hline Masculino & 70,84 & 60,70 & 26,44 & 0,18 \\
\hline Feminino & 70,84 & 68,40 & 19,80 & \\
\hline \multicolumn{5}{|l|}{$3^{\circ}$ e $4^{\circ}$ anos } \\
\hline Masculino & 75,00 & 69,95 & 22,91 & 0,66 \\
\hline Feminino & 66,67 & 67,87 & 19,78 & \\
\hline \multicolumn{5}{|l|}{$5^{\circ}$ e $6^{\circ}$ anos } \\
\hline Masculino & 75,00 & 68,84 & 16,52 & 0,46 \\
\hline Feminino & 58,33 & 64,86 & 19,62 & \\
\hline
\end{tabular}

Meio ambiente

$\begin{array}{ccccc}1^{\circ} \text { e } 2^{\circ} \text { anos } & \text { Mediana } & \text { Média } & \text { DP } & \text { p-valor } \\ \text { Masculino } & 53,13 & 53,20 & 17,75 & 0,16 \\ \text { Feminino } & 62,50 & 58,92 & 15,06 & \\ 3^{\circ} \text { e } 4^{\circ} \text { anos } & & & & \\ \text { Masculino } & 68,75 & 63,64 & 20,56 & 0,30 \\ \text { Feminino } & 59,38 & 59,45 & 12,70 & \end{array}$

$5^{\circ}$ e $6^{\circ}$ anos

$\begin{array}{lllll}\text { Masculino } & 59,38 & 61,82 & 17,42 & 0,16 \\ \text { Feminino } & 53,13 & 54,35 & 18,55 & \end{array}$

Teste $\mathrm{t}$ Student; DP = desvio padrão.

Fonte: Elaborado pelos autores. 
Já em relação ao domínio físico, único em que houve diferença estatística significante, percebe-se uma melhora dos escores no decorrer do curso. No domínio meio ambiente, os estudantes do início e do final do curso obtiveram os piores resultados, embora não haja diferença significativa entre os grupos. Percebe-se que esses indivíduos têm sua capacidade para desempenhar as atividades do dia a dia e o trabalho $(\mathrm{Q} 17=52,42$ e Q18 $=51,37)$ e sua concentração $(\mathrm{Q} 7=56,15)$ prejudicadas, o que pode ser decorrente de uma falta de energia e fadiga intensa $(\mathrm{Q} 10=53,72)$, provocada especialmente pela falta de sono e repouso $(\mathrm{Q} 16=39,97)$.

Cardoso et al. ${ }^{18}$ verificaram que os estudantes de Medicina e residentes têm uma média de horas de sono por noite inferior à da população em geral, o que contribui para uma maior prevalência de sonolência diurna excessiva. Além disso, estudos anteriores demonstraram que os estudantes de Medicina estão suscetíveis a desenvolver a síndrome de burnout, decorrente do intenso esgotamento físico e mental a que estão submetidos durante o processo de formação ${ }^{15}$.

Um estudo realizado com 42 estudantes de Medicina do primeiro ano do Instituto Superior de Ciências Médicas de Camagüey, em Cuba, concluiu que $60,9 \%$ da amostra era vulnerável ao estresse, e os principais eventos responsáveis por causá-lo foram a sobrecarga acadêmica, a falta de tempo para finalizar as atividades e a realização de um exame ${ }^{19}$. Já Querido et al. ${ }^{20}$ evidenciaram que grande parte dos estudantes de Medicina matriculados no internato médico da Universidade Federal de Tocantins (UFT) e do Centro Universitário Tocantinense Presidente Antônio Carlos (Unitpac), localizadas no estado do Tocantins, está sob estresse num estágio potencialmente adoecedor. Embora eles não reconheçam essa condição, destacam a extensa carga horária e o cansaço físico como sendo causadores de estresse.

Tenório et al. ${ }^{21}$, em pesquisa realizada no campus da Universidade Federal de Sergipe (UFS), concluíram que os estudantes de Medicina do curso de modelo "Aprendizado Baseado em Problemas (ABP)", um exemplo de metodologia ativa de ensino, apresentaram estresse, sofrimento e ansiedade em um grau menor daqueles acadêmicos do curso médico de modelo tradicional. A Uncisal, atualmente, segue o modelo flexneriano, o qual se caracteriza pela divisão do curso médico em ciclo básico e ciclo clínico, tendendo, na sua maioria, para o ensino tradicional, centrado em aulas expositivas $^{22}$, o que pode também potencializar o estresse e a fadiga psicológica dos acadêmicos.

Os acadêmicos de Medicina da Uncisal se dedicam, em tempo integral, durante seis anos de sua vida, à graduação; e essa intensa carga horária curricular reflete também na falta de oportunidade de lazer e recreação $(\mathrm{Q} 14=48,94)$ e no quanto o indivíduo aproveita a vida (Q5 $=56,52$ ), e interfere até mesmo na criatividade, pois poucas vezes os estudantes conseguem desviar a sua concentração para assuntos que não tenham relação com a "medicina".

Especialmente por estarem no período da juventude, a restrição do lazer aos futuros médicos compromete a qualidade de vida deles. Em um estudo com acadêmicos de Enfermagem de uma instituição pública do Sul do Brasil identificou a limitação do lazer e o pouco tempo de descanso como as principais causas de estresse no ambiente universitário ${ }^{17}$.

Em relação ao sexo, percebe-se que as mulheres tiveram uma pior avaliação em todos os domínios e nas questões gerais, havendo diferença significativa no domínio psicológico $(\mathrm{p}=0,04)$ e na satisfação com a saúde $(p=0,04)$, entre os alunos do quinto e sexto anos. Meyer et al. ${ }^{23}$ verificaram, em estudo com acadêmicos de Medicina do internato de instituições de Santa Catarina, que os homens apresentam melhor avaliação no domínio psicológico do que as mulheres. Cunha et al. ${ }^{11}$ também observaram, em estudo semelhante, menor escore de qualidade de vida entre as mulheres. As mulheres estão mais suscetíveis a desenvolver transtornos de ansiedade e depressão do que os homens, pois sofrem mais com os estressores psicológicos do dia a dia. Além disso, é também no sexo feminino que mais se reflete acerca da saúde e do significado da vida ${ }^{23,24}$.

\section{CONCLUSÃO}

Avaliar a qualidade de vida dos acadêmicos de Medicina da Uncisal foi de suma importância, especialmente pela grande relevância desse curso em capacitar indivíduos para cuidar da saúde e qualidade de vida da população. O presente estudo pode contribuir para possíveis mudanças que promovam melhorias no processo ensino-aprendizagem, buscando proporcionar uma análise do padrão curricular do curso médico da universidade e desenvolver intervenções capazes de viabilizar o bem-estar dos acadêmicos de Medicina, como a ampliação de serviços de apoio psicopedagógico e acompanhamento sistemático dos discentes.

Essas medidas auxiliarão os discentes a lidar com as dificuldades do curso e buscar saúde física e mental para que possam alcançar melhor qualidade de vida, o que é essencial para o cuidado do outro.

\section{REFERENNCIAS}

1. The Whoqol Group. The World Health Organization Quality of Life assessment (Whoqol): development and general psychometric properties. Soc Sci Med. 1998;46(12):1569-85.

2. Pereira ER, Teixeira CS, Santos A. Qualidade de vida: abordagens, conceitos e avaliação. Rev Bras Educ Fís Esp. 2012;26(2):241-50.

3. The Whoqol Group. The World Health Organization Quality of Life assessment (Whoqol): position paper from the World Health Organization. Soc Sci Med. 1995;41(10):1403-9.

4. Fleck MPA, Louzada S, Xavier M, Chachamovich E, Vieira G, Santos L, et al. Aplicação da versão em português do instrumento abreviado de avaliação da qualidade de vida "Whoqol-Bref”. Rev Saúde Pública. 2000;34(2):178-83.

5. Skevington SM, O'Connell KA, Lotfy M. The World Health Organization's Whoqol-Bref quality of life assessment: psychometric properties and results of the international field trial a report from the Whoqol Group. Qual Life Res. 2004;13: 299-310.

6. Almeida MAB, Gutierrez GL, Marques R. Qualidade de vida: definição, conceitos e interfaces com outras áreas de pesquisa. São Paulo: Escola de Artes, Ciências e Humanidades - Each/USP; 2012.

7. Bampi LNS, Baraldi S, Guilhem D, Araújo MP, Campos ACO. Qualidade de vida de estudantes de Medicina da Universidade de Brasília. Rev Bras Educ Med. 2013;37(2):217-25.

8. Aquino MT. Prevalência de transtornos mentais entre estudantes de medicina da Universidade Federal de Minas Gerais [dissertação]. Belo Horizonte: Universidade Federal de Minas Gerais; 2012

9. Figueiredo AM, Ribeiro GM, Reggiani ALM, Pinheiro BA, Leopoldo GO, Duarte JAH, et al. Percepções dos estudantes de Medicina da Ufop sobre sua qualidade de vida. Rev Bras Educ Med. 2014;38(4):435-43.

10. Alves JGB, Tenório M, Anjos AG, Figueroa JN. Qualidade de vida em estudantes de Medicina no início e final do curso: avaliação pelo 
Whoqol-Bref. Rev Bras Educ Med. 2010;34(1):91-6.

11. Cunha DHF, Moraes MA, Benjamin MR, Santos AMN. Percepção da qualidade de vida e fatores associados aos escores de qualidade de vida de alunos de uma escola de medicina. J Bras Psiquiatr. 2017;66(4):189-96.

12. Moutinho ILD, Maddalena NCP, Roland RK, Lucchetti ALG, Tibiriçá SHC, Ezequiel OS, et al. Depression, stress and anxiety in medical students: a cross-sectional comparison between students from different semesters. Rev Assoc Med Bras. 2017;63(1):21-8.

13. Pedroso B, Pilatti LA, Gutierrez GL, Picinin CT. Cálculo dos escores e estatística descritiva do Whoqol-Bref através do Microsoft Excel. Rev Bras Qual Vida. 2010;2(1):31-6.

14. Saupe R, Nietche EA, Cestari ME, Giorgi MDM, Krahl M. Qualidade de vida dos acadêmicos de enfermagem. Rev Latino-Am Enfermagem. 2004;12(4):636-42.

15. Ramos-Dias JC, Libardi MC, Zillo CM, Igarashi MH, Senger MH. Qualidade de vida em cem alunos do curso de Medicina de Sorocaba - PUC/SP. Rev Bras Educ Med. 2010;34(1):116-23.

16. Tomaschewski-Barlem JG, Lunardi VL, Ramos AM, Silveira RS, Barlem ELD, Ernandes CM. Manifestações da síndrome de burnout entre estudantes de graduação em Enfermagem. Texto \& Contexto Enferm. 2013;22(3):754-62.

17. Hirsch CD, Barlem ELD, Almeida LK, Tomaschewski-Barlem JG, Lunardi VL, Ramos AM. Fatores percebidos pelos acadêmicos de Enfermagem como desencadeadores do estresse no ambiente formativo. Texto \& Contexto Enferm. 2018;27(1):1-11.

18. Cardoso HC, Bueno FCC, Mata JC, Alves APR, Jochims I, Vaz Filho IHR, et al. Avaliação da qualidade do sono em estudantes de Medicina. Rev Bras Educ Med. 2009;33(3):349-55.

19. Martín YD. Estrés académico y afrontamiento en estudiantes de Medicina. Rev Hum Med. 2010;10(1) [citado em 28 jul 2018]. Disponível em: http://scielo.sld.cu/pdf/hmc/v10n1/hmc070110.pdf.

20. Querido IA, Naghettini AV, Orsini MRCA, Bartholomeu D, Montiel JM. Fatores associados ao estresse no internato médico. Rev Bras Educ Med. 2016;40(4):565-73.
21. Tenório LP, Argolo VA, Sá HP, Melo EV, Costa EFO. Saúde mental de estudantes de escolas médicas com diferentes modelos de ensino. Rev Bras Educ Med. 2016;40(4):574-82.

22. Pagliosa FL, Da Ros MA. O Relatório Flexner: para o bem e para o mal. Rev Bras Educ Med. 2008;32(4):492-9.

23. Meyer C, Guimarães ACA, Machado Z, Parcias SR. Qualidade de vida e estresse ocupacional em estudantes de Medicina. Rev Bras Educ Med. 2012;36(4):489-98.

24. Jasen K, Mondin TC, Ores LC, Souza LDM, Konradt CE, Pinheiro $\mathrm{RT}$, et al. Transtornos mentais comuns e qualidade de vida em jovens: uma amostra populacional de Pelotas, Rio Grande do Sul, Brasil. Cad Saúde Pública. 2011;27(3):440-8.

\section{CONTRIBUIÇÃO DOS AUTORES}

Aline Maria Fatel da Silva Pires participou como bolsista do Programa Institucional de Bolsas de Iniciação Científica da Fundação de Amparo à Pesquisa do Estado de Alagoas (Pibic/Fapeal) e contribuiu com a idealização e elaboração do projeto e com a coleta dos dados, escreveu o artigo e revisou a versão final. Waléria Dantas Pereira Gusmão foi a professora orientadora, contribuiu com a idealização e elaboração do projeto e com a coleta dos dados, escreveu o artigo e revisou a versão final. Monique Maria Lucena do Suruagy do Amaral participou da elaboração do projeto e o submeteu ao Comitê de Ética em Pesquisa. Lucyo Wagner Torres de Carvalho realizou a análise estatística.

\section{CONFLITO DE INTERESSES}

Os autores declaram não haver conflito de interesses neste estudo.

\section{ENDEREÇO PARA CORRESPONDÊNCIA}

Waléria Dantas Pereira Gusmão. Avenida Aristeu de Andrade, 492, Edifício Giardino de Mare, ap. 502, Farol, Maceió, AL, Brasil. CEP: 57051-090. E-mail: waleriadantasnut@gmail.com 